\title{
Design of Intelligent Transportation Inquiry System Based on MapX in the Environment of VC++
}

\author{
Juan Cheng ${ }^{1}$ and Lu Sun ${ }^{2}$ \\ ${ }^{1}$ School of Transportation, Southeast University, No.2 Sipailou, Nanjing, China \\ ${ }^{2}$ Department of Civil Engineering, Catholic University, Washington, DC, US
}

\begin{abstract}
This paper applied MapInfo, the professional soft ware tool of GIS, integrated secondary exploiture combining with electronic maps, and made use of the exploiture flat roof Visual $\mathrm{C}++$ as the tool of visualize development, transferred MapX, a control of MapInfo, integrated them. The paper designed the Inquiry System in Intelligent Transportation, which including query system of road information, query system of bus information, query system of district information. It can be carried out space analysis and query function based on GIS. Adopted SQL Server manage attribute data, by data binding, attribute data in SQL Server and victor picture data were combined.
\end{abstract}

\section{Introduction}

Transportation is the link and lifeline of urban economic activity, which plays an important role on the sustainable development of city and the improvement of people's living standards. With the rapid development of the national economy, in many cities of the world the phenomenon of traffic congestion appeared which caused the traffic accident, traffic noise, traffic pollution and so on. In order to improve the traffic condition in a region, the intelligent transportation system (ITS) has emerged. ITS is a comprehensive, real-time, accurate, safe and efficient transportation system with advanced electronic technology, information technology, sensor technology and system engineering technology [1]. Geographical Information System (GIS) is an important part of the intelligent transportation system, which is based on its outstanding spatial information management capability.

\section{Overview of related technologies}

\subsection{Geographical Information System for Transportation (GIS-T)}

GIS-T is a computer software and hardware system for collecting, storing, managing, analyzing, processing spatial information and traffic information, which is the extension of GIS in the traffic field. In a word, GIS-T is an integrated technology of GIS for transportation [2].
SQL Server is a relational database management system for $\mathrm{C} / \mathrm{S}$ model [3]. SQL Server is data storage and retrieval model in the middle of 1980 s and has continued to develop to nowadays, which tightly integrate with Windows NT operating system [4].

\section{Design of intelligent transportation inquiry system}

\subsection{System requirement analysis}

Intelligent transportation information inquiry system using graphics display function of GIS is a graphical operating platform, which provides users with interface who can easily query and management [5], [6].

This paper uses Visual $\mathrm{C}++$ and MapX to develop, using MS SQL Server as background database and using SQL language through the ADO database access interface to control the database.

\subsection{Overall design of system function}

\subsubsection{Overall system}

The system which makes full use of the map display function of GIS displays the different geographical elements and supports the management and function of data.

\subsubsection{System requirements}

(1) The system operation must be simple and practical. 
(2) Security of information system.

(3) Stability and reliability of the system.

\section{Realization of the system function}

\subsection{Realization of the system database}

The composition of the database of intelligent transportation information inquiry system is shown in Fig. 1.

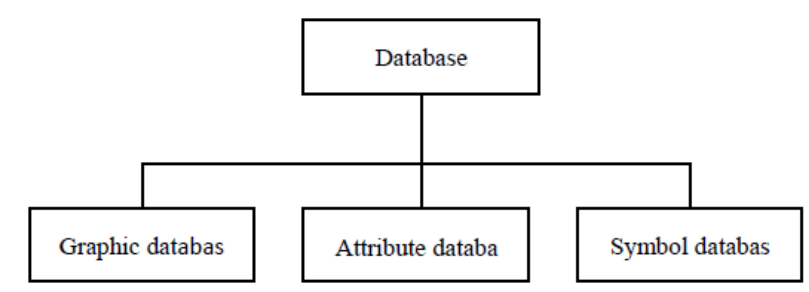

Figure 1. the Construction of the System Database Diagram

Database table is an important part of the system. During the formation of each map layer, MapInfo create a database table for each layer automatically. Processing of map layer by MapInfo is actually a map layer that corresponds to a database table processing [7].

\subsubsection{Graphic database}

Graphics database of intelligent transportation information inquiry system is scanned according to the map obtained, which can only be used as a base map. Other layers are obtained by two methods. One is the use of AutoCAD, which is shown in Fig. 2. Another, it should draw a map layer with MapInfo when the system requires a new layer. MapInfo will generate database table and the corresponding file automatically.

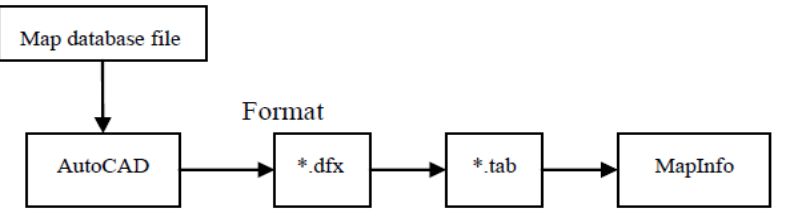

Figure 2. Format conversions of MapInfo and AutoCAD diagram

\subsubsection{Attribute database}

The relational data structure is used in the MapInfo attribute database. The attribute database of main layers of the system is shown in Table 1.

where:

RoadId is the number of the road.

RoadName is the name of the road.

RoadLength is the length of the road.

RoadWidth is the width of the road.

RoadRank is the level of the road.

Int is integer. The storage size of this type is 4 bytes and the range of the value is $-2^{31} \sim 2^{31}-1$.

Varchar is character and the format is varchar[(n)].
The storage size is the actual length of the input character.

Float is floating-point and the format is float $[(\mathrm{n})] . \mathrm{N}$ is used to store the mantissa of scientific notation where the range of the value is $1 \sim 53$.

Table 1. Structure of road information table

\begin{tabular}{l|l|lll|} 
name of the rov & date troe & length|allov nuld \\
\hline RoadId & int & 4 & \\
Roadiane & varchar & 10 & $\checkmark$ \\
RoadLength & float & 8 & $\checkmark$ \\
Roadridth & float & 8 & $\checkmark$ \\
RoadRank & varchar & 10 & $\checkmark$ \\
& & &
\end{tabular}

\subsubsection{Symbol database}

MapInfo system has its own symbol database shown in Fig. 3.

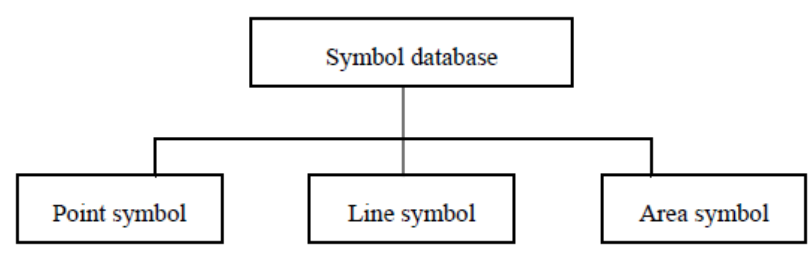

Figure 3. The symbol database of system diagram.

\subsection{Realization of the system function}

\subsubsection{Security module}

The system is set up a table to store user names and passwords in the database. The system compares the user name and password entered by the user with the information existed in the database, if the user exists and the password is correct, then enter the main interface. Landing fenster display error messages when user name or password is incorrect.

User types are divided into system administrators and ordinary users. The system administrator can change the administrator's password and modify information of general user, while ordinary users can modify their own user name and password.

User table structure is shown in Table 2.

Table 2. Structure of User Table

\begin{tabular}{|c|c|c|c|}
\hline USERID & USERNAME & USERPWD & USERTYPE \\
\hline 1 & admin & $* * * * * * *$ & $\begin{array}{l}\text { system } \\
\text { administrator }\end{array}$ \\
\hline 2 & april & $* * * * * * *$ & ordinary users \\
\hline 3 & fly & $* * * * * * *$ & ordinary users \\
\hline$\ldots \ldots$ & $\ldots \ldots \ldots$ & $\ldots \ldots$. & $\ldots \ldots$ \\
\hline
\end{tabular}

In order to protect the security of the database, in addition to setting password, we take a database backup 
function. When the destruction in the database, we can restore the database.

\subsubsection{Operation module}

(1) Basic Operations Modulz

Basic operations module includes arrows, zoom in, and zoom out, pan, full map display, central display. These functions can be found on the system function navigation bar

(2) Advanced Operations Module

Advanced operations module includes selection, rectangular selection, round selection, polygon selection and ranging

(3) Inquiry Module

- Road information inquiry

We can inquiry the length, width and grade of the road. We can modify information of each road and save the changes to the database at the same time.

- Traffic districts information inquiry

We can inquiry the range, area and population of the traffic zone. We can modify information of each traffic zone and save the changes to the database at the same time.

- Bus information inquiry

We can inquiry peak headway, flat peak headway, early morning departure time, evening departure time, fares, starting station and terminus of each line. We can modify information of each line and save the changes to the database at the same time.

- Optimal path algorithm of least transfer

According to travel habits of people, we choose the bus immediately if there is a direct line from station A to station B. If there is more than one direct line, then considering the route of the distance, we choose the nearest bus scheme. If there is no direct bus, we will consider changing a bus. If there is a station $\mathrm{C}$ which is the public station of station $\mathrm{A}$ and station $\mathrm{B}$, we will change the bus at station $C$. If there is no station $C$, we will consider changing a bus in station $D$ which is the public station of station $\mathrm{C}$ and station $\mathrm{B}$. If there is no station $\mathrm{D}$, then we will change bus more than three times to the destination. If there is more than one choice in the above case, we will consider the distance and will choose the shortest travel plan.

Transfer plans of bus lines are shown in Figure 4.

- Shortest Path Analysis

Determine bus changing plan of the shortest path between the two places of city.

(4) Analysis Module

- Road information inquiry

The system shows the number of all main road and inquiries the length of the each main road which can navigate to the corresponding location in the map.

- Positioning traffic districts

Select a traffic zone, the system can be positioned to the map and highlight it.

- Ratio of main road, secondary road and branch road in urban road network

$$
\gamma=\sum_{i=1}^{m} a_{i}: \sum_{j=1}^{n} b_{j}: \sum_{k=1}^{p} c_{k}
$$
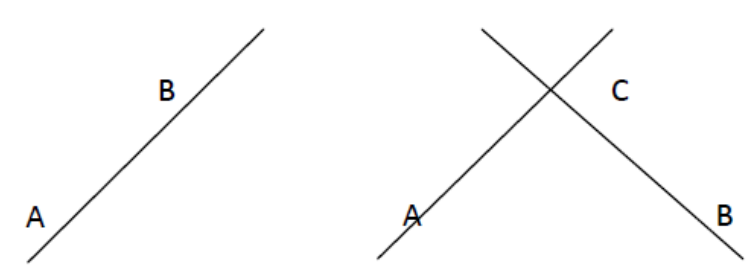

Direct line

Change one bus
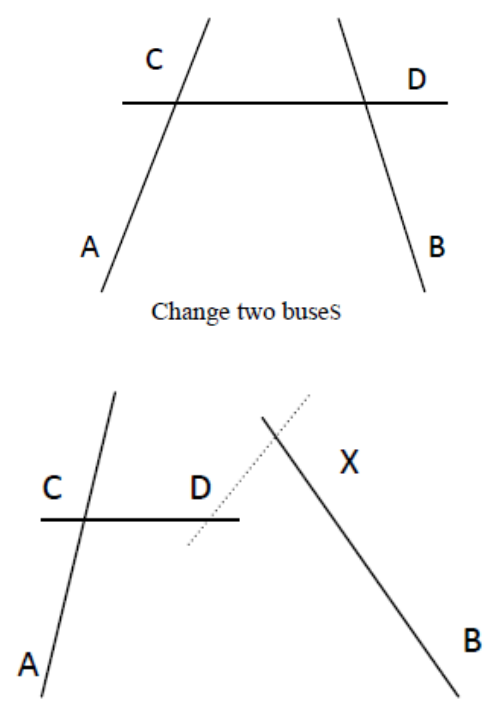

Change multiple buses

Figure 4. Transfer plans of bus lines.

In equation (1), $\gamma$ is the ratio of main road, secondary road and branch road in urban road network. $a_{i}$ is the mileage of main road in road network where $i=0,1,2, \ldots, \mathrm{m}$. $b_{j}$ is the mileage of secondary road in road network where $\mathrm{j}=0,1,2, \ldots, n$. $\mathrm{c}_{\mathrm{k}}$ is the mileage of branch road in road network where $\mathrm{k}=0,1,2, \ldots, \mathrm{p}$.

- Thematic Map

For a large number of digital data, the user may not value the specific figures, but want to get the overall distribution of the situation, For example, population density thematic map. If users need to get the distribution of the total number of male and female population in each area, they can choose the number of male or female population as a special variable and create a pie chart, etc.

\section{Example}

According to the method above, Intelligent Transportation Inquiry System of $\mathrm{X}$ city is constructed in this paper. Intelligent Transportation Inquiry System of Nanchang city uses Visual $\mathrm{C}++$ and MapX to develop, using MS SQL Server as background database and using SQL language through the $\mathrm{ADO}$ database access interface to 
control the database. The main functions of the system are shown as follows.

- Login System Diagram is shown in Fig. 5.

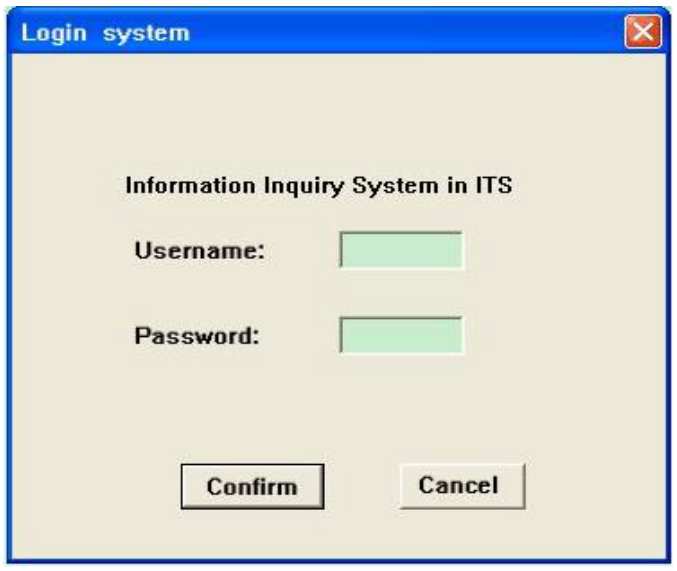

Figure 5. Login system diagram.

-Main Operating Diagram is shown in Fig. 6.

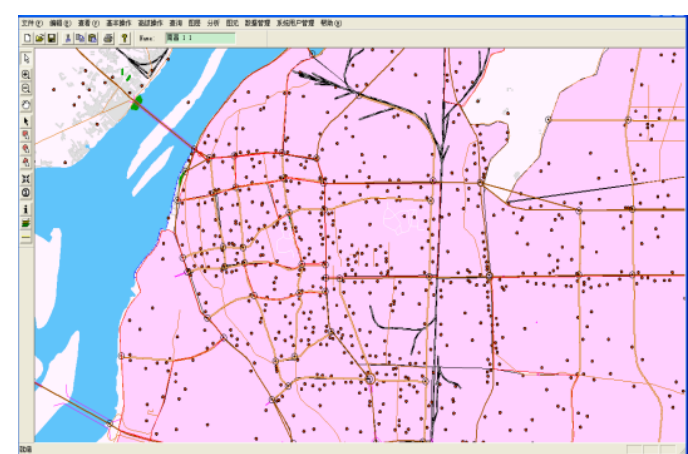

Figure 6. Main operating diagram

\section{Conclusion}

This paper describes the traffic geographic information system and database technology, and also introduces the function design of the intelligent transportation inquiry system in detail. Using Visual $\mathrm{C}++$ programming language combined with GIS component MapX and Server SQL database, this article developed intelligent transportation inquiry system and completed each module of the system. Finally, the paper users Intelligent Transportation Inquiry System of Nanchang city to verify.

\section{Acknowledgment}

This study is sponsored in part by the National Science Foundation under Grants CMMI-0408390 and CAREER award CMMI-0644552, by the National Natural Science Foundation of China under grants 51250110075, 513111130and U1134206, by Ministry of Communication of China under grant $0901005 \mathrm{C}$, and by Jiangsu Natural Science Foundation under Grant SBK200910046, to which the authors are very grateful. The authors would also like to thank anonymous reviewers for their very helpful comments and constructive suggestions, with help us to improve the content and presentation of the manuscript.

\section{References}

1. National ITS Architecture for America (Version 4.0), CD-ROM, (2002).

2. S. B. Miles, Application and Issues of GIS as Tool for Civil Engineering Modeling, Journal of Computing in Civil Engineering, 13(3): 144-152, (1999).

3. H. J. Miller, Yi-Hwa Wu, GIS Software for Measuring Space-Time Accessibility in Transportation Planning and Analysis, GeoInformatica, 4(2), 141-159, ( 2000).

4. R. Beheshti, R. Miehels., The global GIS: a ease study, Civil Engineering Informatics, Faculty of Civil Engineering and Geosciences, Delft University of Technology, Netherlands, (2001).

5. Jianjun Xiao, Research and application of Transportation Geographic Information System, Central South University, (2005).

6. F. Kurt, W. Lotharand, A Hybrid Expert System, GIS and Simulation Modeling for Environmental and Technological Risk Management, Computer-aided Civil and Infrastructure Engineering, (1999).

7. D Xiong, H Lin, Spatial Data Handling for ITS: Perspective, Issues and Approaches, GeoInformatica, 4(2), 215-230, (2002). 